\title{
Cell as a Living System
}

\author{
Martin Vlček* \\ e-Functionality, Non-profit research group, Czech
}

*Corresponding author: Martin Vlček, eFunctionality, non-profit research group, Prague, Czech Republic

To Cite This Article: Martin Vlček. Cell as a Living System. 2020 - 9(3). AJBSR.MS.ID.001390. DOI: 10.34297/AJBSR.2020.09.001390.

Received: 㘹: April 28, 2020; Published: 眥 June 30, 2020

\section{Introduction}

Results of model work on human systems are also used to understand the cell system. The principle of the living system is extended by a more or less suspected structure of dark matter. Dark matter as a rather astronomical term is interpreted as a fundamental part of the structure of living systems. Its function is connected with the materialization and control of the concept of existence.

\section{Living Systems}

Living systems evaluate their existence on the basis of the satisfaction of their elements, and therefore on their (migratory) tendency to leave the system - the object. The degree of tendency is, in a certain simplification, the degree of existence.

The living object constantly evaluates the development of its own existence, and if it identifies an event known to it that announces the decline of existence, it activates the systemic means of reconstruction. They first evaluate the distribution of activities in the system and then activate the reconstruction part. As a result of its activities, there will be a change in the distribution of satisfaction and thus the required change in the existence of the object [1].

However, the living system thus described is only a part of it in normal matter. Dark matter then realizes its connection with other objects, in the realm of existence. Above all, due to dark matter, the living object is activated in the evaluation of its existence. The evaluation of the existence of a living object occurs only in times that are defined by dark matter, no universal time is available . Dark matter at this time acts as a pace-maker.

The feedback to dark matter is then the existence of one's own living system, which occurs when the object as a whole is activated. At the same time, the distribution of success and the reconstruction of relationships are evaluated in such a way that the stress in satisfaction is also solved by reducing the stress in normal matter.

The object exists at the moment when its structure is activated in normal matter and also at this moment satisfaction is defined at all its levels. It is defined through this structure, and dark matter, through the abilities of its parts, is able to identify it [2].

\section{Cell System}

The general scheme of the origin of any living system is as follows. In the first phase, these are cooperating elements for which cooperation is more advantageous than independence. In the case of the cell, it is a precursor of mitochondria, a kind of precursor of today's bacteria. So far, their relationships are based on the advantages of mutual relationships, and everything takes place in normal matter. Gradually there is a specialization.

Within it, a group is formed that specializes in activities that are influenced not by observable events in normal matter, i.e. by the exchange and success of individual elements, but by their satisfaction. This is generally reflected in their tendency to leave the emerging system. These elements of the group thus gradually fall out of the normal world (matter) and approach dark matter. Their activity is a function of satisfaction - i.e. existence and from the point of view of others in the group they become dead.

Because it is a group that specializes in generally needed activity, as they gradually die, they seem to surround the rest with their "torso". Alternatively, at least the rest permeate it. The group probably solves its function - monitoring migration and thus also its existence - in some cases by somehow limiting the movement of normal matter - other elements.

The torso - in the case of a cell cytoplasm with a cell wall and organelles - consists of the "remains" of dead elements and especially of their structure evaluating the existence. This structure thus takes its function from the original elements, it is an evaluation of existence and it is in cooperation with dark matter. This crucial structure in the case of a cell is based on elements one level lower and these are the water molecules and its structures. More specifically, these are hydroxonium structures and dynamic hydrogen bonds. 
Here it is based on the assumption that the original bacteria mitochondria - are similarly formed, as a living object above water as its elements. The result is a living system whose building blocks - the elements - are mitochondria, which move in a "torsion of dead" mitochondria. This torso - the cytoplasm - is gradually evolving and improving, but still only with the task of being able to better evaluate the "satisfaction" of the elements - mitochondria.

\section{Conclusion}

Since the assumption of unity of not only material but also principles in different levels of organization of matter in mutual cooperation of normal and dark matter is made, it is possible to ask how transferable are possible experiences, procedures, events, intelligence between levels in general [3].

If so, it would be interesting to observe and get to know more distant systems and understand their life principles and use them in systems such as humans. This would probably be more useful than examining systems as close as possible, which are the easiest to understand but all the less beneficial.

However, it is probable that the interconnectedness of the existence of various living systems through dark matter is, among other things, the transfer of this intelligence between levels with a single goal - maximizing global existence.

\section{Reference}

1. Martin Vlček (2018) Dark Matter in our Ordinary Life. Amazon in Wroclaw.

2. Martin Vlček (2020) The Existence. In Clinical Reviews \& Cases.

3. Martin Vlček (2020) Virus as a Living Object. In American Journal of Virology and Diseases. 